\title{
Plants in the Songbooks of Castilla y León, Spain
}

\author{
M. Cardaño and B. Herrero
}

\section{Research}

\begin{abstract}
Man's relationship with plants is reflected in cultural manifestations such as music. This work aims to present the most important plants in the traditional songs of Castilla y León, Spain. Thirty-three publications were reviewed, from which 7012 songs collected from the oral tradition were transcribed and information about the botanical quotations in these songs was extracted. Plant species were listed in 1316 songs, and 2756 botanical quotes were collected, alluding to 150 species. The most frequently mentioned plants were rose $(12.7 \%)$, grape $(7.1 \%)$, carnation $(6.3 \%)$, and wheat $(5.1 \%)$. In $32.7 \%$ of the citations, the use of the plants could be deduced, and in $64.6 \%$ of the citations, the plant organ alluded to was determined. The most frequently mentioned plants symbolize values of beauty or they are plants of significant economic value in the region of study. Food and decoration are the uses most frequently mentioned, the fruit or flower being alluded to most in the songs.
\end{abstract}

\section{Resumen}

La relación del hombre con las plantas alcanza manifestaciones culturales como la música. Este trabajo tiene por objeto presentar las plantas más importantes en las canciones tradicionales de Castilla y León. Se revisaron 33 publicaciones donde están transcritas 7.012 canciones que han sido recogida de la tradición oral, y se extrajo la información sobre las citas botánicas que se encuentran en dichas canciones. En 1.316 canciones se mencionan especies vegetales, se recogieron 2.756 citas botánicas aludiendo a 150 especies. Las especies más mencionadas fueron: rosa $(12,7 \%)$, vid $(7,1 \%)$, clavel $(6,3 \%)$, y trigo $(5,1 \%)$. En el $32,7 \%$ de las citas se dedujo el uso que tenían las plantas y en el $64,6 \%$ de las menciones se determinó el órgano vegetal al que se aludía. Las plan- tas más mencionadas simbolizan valores de hermosura y belleza o plantas de significativo valor económico en la región de estudio. El uso alimentario o decorativo son los más mencionados aludiendo en las canciones al fruto o a la flor.

\section{Introduction}

Ethnobotany is an integrative, multi-disciplinary field of learning. One of the many branches of investigation that draws on cultural knowledge and that begins with the prefix "ethno" is ethnomusicology, the study of the music and dance of different cultures and the musical instruments those cultures make and use, which are often made of plant materials. The use of plants to produce musical instruments has been addressed in several studies (Keller 2010, Puri \& Chaturvedi 2008).

Ethnobotany is the science that describes the relationship between human and plants, but the factors that influence that relationship are not well defined. The interdisciplin-

\section{Correspondence}

M. Cardaño and B. Herrero, Departamento de Ciencias Agroforestales, Universidad de Valladolid, Avenida de Madrid 57, 34004 Palencia, SPAIN. baudilio@agro.uva.es

Ethnobotany Research \& Applications 12:535-549 (2014)

Published: 24 November 2014 
ary approach is well known in ethnobotany (Etkin \& Elisabetsky 2005) and can help us to understand what factors may interact and have a decisive influence on the selection of plants used by humans (Akerreta et al. 2007).

Thus the tools of ethnobotanical investigations are many: botany, mycology, taxonomy, anthropology, ethnography, archaeology, comparative folklore, religious studies, medicine, chemistry, and pharmacology (Harris 2008, Holman 2002). Sometimes, in ethnobotanical inquiry, we call upon ancient history, or colonial socio-economic histories, or we even examine the roots of our modern social movements.

Studies on plants in musical compositions are scarce; one example is the study by Causapé and De la Torre (2009) on plants in a Christmas carol. References to plants in significant texts such as the Bible and works of Cervantes have also been analyzed (Moldenke \& Moldenke 2002, Pardo de Santayana et al. 2006).

The aim of this paper is to highlight the importance of plants in cultural manifestations such as music, to determine which plants form part of the musical stanzas, which plant has a greater presence in the songs of Castilla $y$ León, which uses are attributed to these plants in the musical texts, and which parts of the plants are referenced.

\section{Material and Methods}

A search of the folk songs of the region was carried out. Thirty-three popular songbooks were located, representing the musical works of provinces, districts, and towns located in the regional context of Castilla y León. The musical works include verses as text, not musical scores. These works are listed in Appendix I.

The authors of the songbooks have collected the songs according to ethnographic methodology, transcribing into written texts the songs that have been passed down by oral tradition over the centuries.

The following sources were consulted to attain the songbook material: the library archives of the Faculty of Arts at the University of Valladolid, the provincial archive of Palencia, the Joaquín Díaz Foundation Library in Urueña (Valladolid), the Network of Libraries of Castilla y León, and internet document searches.

The various songs compiled in the songbooks were reviewed one by one, noting the following details: town, province, song title, song type, time of year represented, the fragment of the song where plant species appear, any by-products mentioned (such as white or black bread or wine), and the context. With this information the data were organized in the following fields: the common name of the plant mentioned, the number of times it is cited in a song, the part of the plant referred to, and the use or uses of the plant in the text. Assignment to the categories of use was carried out according to the criteria of the Iberian ethnobotanical group (Pardo de Santayana 2008).

To relate the common name with the scientific name, a number of publications were used (Ceballos Jiménez 1986, Morales et al. 1996, Sánchez 1991). In the case of common names that relate to various botanical species, we chose to assign the most common plant in the region, and in some cases we chose to associate the common name with a scientific genus since we were not able to add further detail. The scientific names of the plants were then updated to the currently accepted names listed in public taxonomic databases (The Plant List 2013, Tropicos 2014). Hereafter we use the term "species" understanding that in some cases we also speak of several species of one genus. Also, for each plant referenced, the status of presence and life-form were noted following the criteria of Mateo and Crespo (1995) and Ruiz de la Torre (2006).

If the same song appeared in several locations, the songs were merged into one record. When the verses alluded to more than one use of a plant, we recorded as many citations as uses mentioned.

\section{Area of study}

Castilla y León spans a total area of $94,147 \mathrm{~km}^{2}$. It is situated in the northern Iberian Peninsula, in southwestern Europe. It is a scarcely populated territory. Half of its 2.6 million inhabitants live in rural settlements. It is therefore a rural society whose economy is maintained by agriculture and natural resources.

Being an inland region, Castilla y León experiences irregular rains and drought during the summer, corresponding to a Mediterranean climate. However, the region also includes a Mediterranean forest dominated by Quercus ilex L. and Quercus faginea Lam. In northern outlying mountainous areas, an Atlantic clime is found, with alpine conditions at the highest elevation. In these conditions one finds an Atlantic forest dominated by Fagus sylvatica $L$. and Quercus petraea (Matt.) Liebl. Rainfalls range from 400 to $1500 \mathrm{~mm} /$ year. Nearly $40 \%$ of the land is devoted to crops while $25 \%$ is covered by pastures and $30 \%$ by woodlands (Cardaño 2009).

\section{Results}

Thirty-three publications with a combined total of 7012 songs were reviewed and analyzed, yielding 1316 songs $(19 \%)$ that mentioned plants in their verses. These songs contained 2756 references to plant species. Figure 1 shows the number of plants that are cited in the songs. The maximum number of plants mentioned in one song was 17 , corresponding to a wedding song from Alcozar 


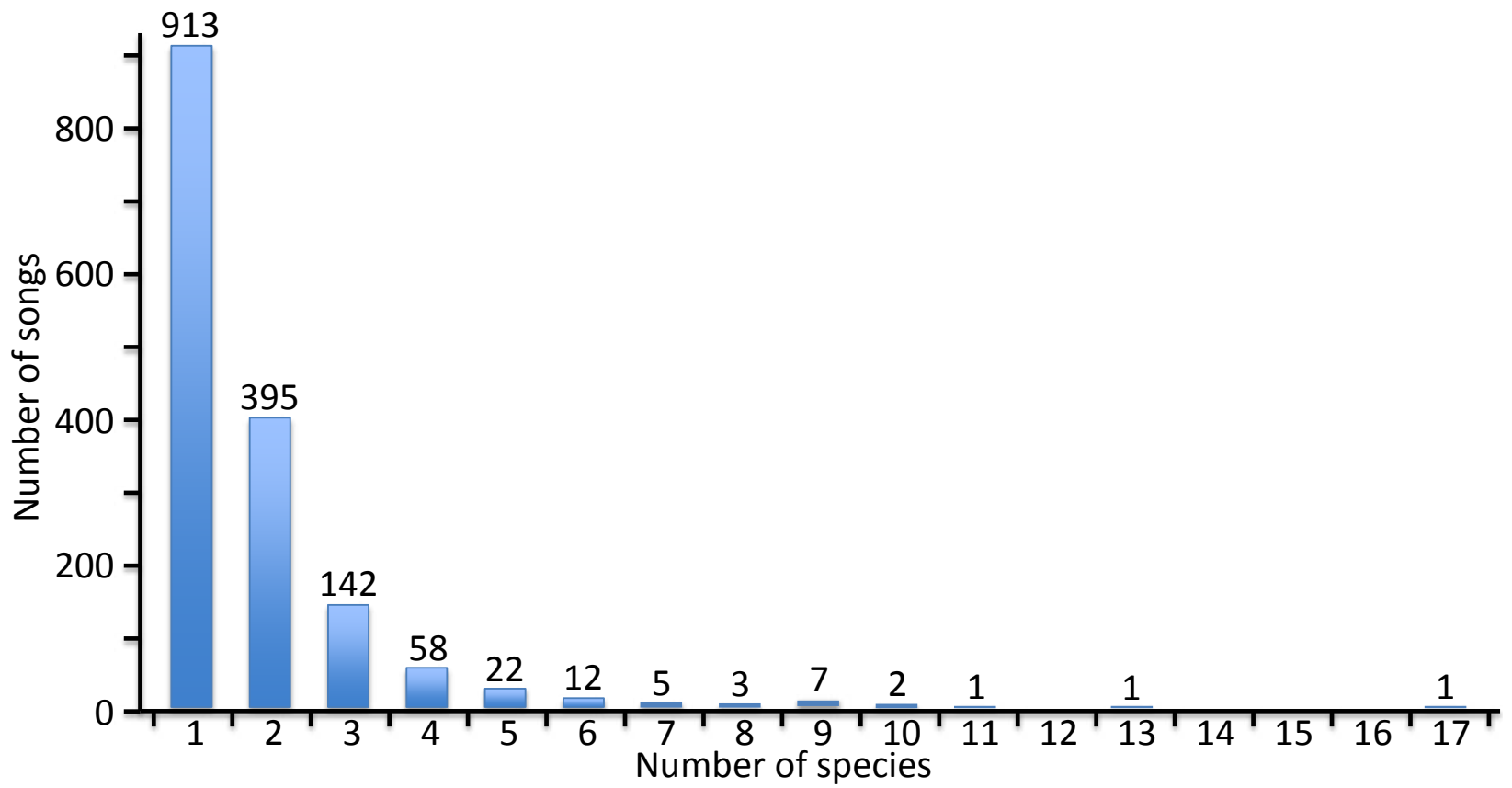

Figure 1. Distribution of the songs of Castilla y León, Spain, according to the number of plants mentioned.

(Soria). This song mentions the following plant species: palm, olive, wheat, mint, parsley, grape, flax, rosemary, blackthorn, elm, lavender, rose, turnip, carnation, tobac$\mathrm{co}$, cocoa, and hazelnut. Of the songs mentioning plants, a majority $(59 \%)$ referenced a single species, with $26 \%$ mentioning 2 species.
Table 1 shows the 150 plants mentioned in the songs. Fifty-five species had more than 10 citations. The most mentioned plants were rose (Rosa spp.), grape (Vitis vinifera L.), carnation (Dianthus caryophyllus L), and wheat (Triticum spp.).

Of the plants mentioned, $43.2 \%$ are cultivated crops, $38.6 \%$ are wild species, $10 \%$ are absent (they are not rep-

Table 1. Plants mentioned in songbooks of Castilla y León, Spain.

\begin{tabular}{|c|c|c|c|c|}
\hline Scientific name & Local name & Citations & Presence & Life-form \\
\hline \multicolumn{5}{|l|}{ Amaranthaceae } \\
\hline Beta vulgaris $\mathrm{L}$. & remolacha & 3 & crop & hemicryptophyte \\
\hline \multicolumn{5}{|l|}{ Amaryllidaceae } \\
\hline Allium ampeloprasum L. & puerro & 9 & crop & geophyte \\
\hline Allium cepa L. & cebolla & 14 & crop & geophyte \\
\hline Allium sativum $\mathrm{L}$. & ajo & 24 & crop & geophyte \\
\hline Allium schoenoprasum L. & cebollino & 1 & crop & geophyte \\
\hline \multicolumn{5}{|l|}{ Apiaceae } \\
\hline Cuminum cyminum L. & comino & 2 & wild & hemicryptophyte \\
\hline Daucus carota L. & zanahoria & 2 & crop & hemicryptophyte \\
\hline Eryngium campestre L. & cardo corredor & 1 & wild & hemicryptophyte \\
\hline Petroselinum crispum (Mill.) Fuss & perejil & 40 & crop & hemicryptophyte \\
\hline Pimpinella anisum L. & anís & 3 & wild & hemicryptophyte \\
\hline \multicolumn{5}{|l|}{ Araliaceae } \\
\hline Hedera helix L. & hiedra & 11 & wild & phanerophyte \\
\hline \multicolumn{5}{|l|}{ Arecaceae } \\
\hline Phoenix dactylifera L. & palmera & 18 & absent & phanerophyte \\
\hline
\end{tabular}




\begin{tabular}{|c|c|c|c|c|}
\hline Scientific name & Local name & Citations & Presence & Life-form \\
\hline Roystonea regia (Kunth) O.F.Cook & palma real & 1 & absent & phanerophyte \\
\hline \multicolumn{5}{|l|}{ Asparagaceae } \\
\hline Asparagus officinalis L. & espárrago & 1 & crop & geophyte \\
\hline \multicolumn{5}{|l|}{ Asteraceae } \\
\hline Anthemis spp.; Matricaria spp. & manzanilla & 3 & wild & therophyte \\
\hline Bellis perennis L. & margarita & 4 & wild & hemicryptophyte \\
\hline Calendula arvensis M.Bieb. & maravilla & 2 & wild & therophyte \\
\hline Carduus spp.; Cirsium spp. & cardo & 4 & wild & hemicryptophyte \\
\hline Cichorium endivia L. & escarola & 1 & crop & hemicryptophyte \\
\hline Cichorium intybus L. & achicoria & 1 & wild & hemicryptophyte \\
\hline Cynara scolymus L. & alcachofa & 1 & crop & hemicryptophyte \\
\hline Dahlia $\times$ hortensis Guillaumin & dalia & 1 & decorative & geophyte \\
\hline Helichrysum stoechas (L.) Moench & siempreviva & 2 & wild & chamaephyte \\
\hline Lactuca sativa L. & lechuga & 28 & crop & hemicryptophyte \\
\hline \multicolumn{5}{|l|}{ Betulaceae } \\
\hline Corylus avellana L. & avellano & 45 & wild & phanerophyte \\
\hline \multicolumn{5}{|l|}{ Brassicaceae } \\
\hline Brassica napus L. & nabo & 13 & crop & chamaephyte \\
\hline \multirow[t]{3}{*}{ Brassica oleracea L. } & berza & 1 & crop & chamaephyte \\
\hline & coliflor & 4 & crop & chamaephyte \\
\hline & repollo & 8 & crop & chamaephyte \\
\hline Erysimum $\times$ cheiri (L.) Crantz & alhelí & 7 & decorative & chamaephyte \\
\hline Nasturtium officinale R.Br. & berro & 7 & wild & hemicryptophyte \\
\hline \multicolumn{5}{|l|}{ Burseraceae } \\
\hline Boswellia sacra Flueck. & incienso & 1 & absent & phanerophyte \\
\hline Commiphora myrrha (Nees) Engl. & mirra & 1 & absent & phanerophyte \\
\hline \multicolumn{5}{|l|}{ Cannabaceae } \\
\hline Cannabis sativa $\mathrm{L}$. & cáñamo & 3 & crop & phanerophyte \\
\hline \multicolumn{5}{|l|}{ Caryophyllaceae } \\
\hline Dianthus caryophyllus L. & clavel & 173 & crop & hemicryptophyte \\
\hline \multicolumn{5}{|l|}{ Cistaceae } \\
\hline Cistus spp. & jara & 1 & wild & phanerophyte \\
\hline \multicolumn{5}{|l|}{ Cucurbitaceae } \\
\hline Citrullus lanatus (Thunb.) Matsum. \& Nakai & sandía & 6 & crop & therophyte \\
\hline Cucumis melo L. & melón & 18 & crop & therophyte \\
\hline Cucumis sativus L. & pepino & 11 & crop & therophyte \\
\hline Cucurbita pepo L. & calabaza & 39 & crop & therophyte \\
\hline \multicolumn{5}{|l|}{ Cupressaceae } \\
\hline Cupressus sempervirens L. & ciprés & 5 & decorative & phanerophyte \\
\hline \multicolumn{5}{|l|}{ Cyperaceae } \\
\hline Schoenoplectus lacustris (L.) Palla & junco marino & 1 & wild & geophyte \\
\hline
\end{tabular}




\begin{tabular}{|c|c|c|c|c|}
\hline Scientific name & Local name & Citations & Presence & Life-form \\
\hline \multicolumn{5}{|l|}{ Ericaceae } \\
\hline Arbutus unedo L. & madroño & 3 & wild & phanerophyte \\
\hline \multicolumn{5}{|l|}{ Fabaceae } \\
\hline Arachis hypogaea L. & cacahuete & 1 & crop & therophyte \\
\hline Ceratonia siliqua L. & algarrobo & 2 & decorative & phanerophyte \\
\hline Cicer arietinum L. & garbanzo & 13 & crop & therophyte \\
\hline Cytisus scoparius (L.) Link & retama & 19 & wild & phanerophyte \\
\hline Genista florida L. & piorno & 1 & wild & phanerophyte \\
\hline Genista spp. & aulaga & 3 & wild & phanerophyte \\
\hline Lathyrus sativus L. & almorta & 6 & crop & therophyte \\
\hline Lens culinaris Medik. & lenteja & 7 & crop & therophyte \\
\hline Lupinus albus L. & altramuz & 1 & wild & therophyte \\
\hline Phaseolus vulgaris L. & judía & 10 & crop & therophyte \\
\hline Pisum sativum L. & guisante & 3 & crop & therophyte \\
\hline Tamarindus indica L. & tamarindo & 1 & absent & phanerophyte \\
\hline Trifolium spp. & trébol & 12 & wild & hemicryptophyte \\
\hline Ulex europaeus L. & tojo & 2 & wild & phanerophyte \\
\hline Vicia ervilla Medik. & yero & 1 & crop & therophyte \\
\hline Vicia faba $\mathrm{L}$. & haba & 16 & crop & therophyte \\
\hline Vicia hirsuta (L.) Gray & arveja & 1 & wild & therophyte \\
\hline & legumbre & 1 & & \\
\hline \multicolumn{5}{|l|}{ Fagaceae } \\
\hline Castanea sativa Mill. & castaño & 25 & wild & phanerrophyte \\
\hline Quercus faginea Lam. & roble carrasco & 1 & wild & phanerrophyte \\
\hline Quercus ilex L. & encina & 31 & wild & phanerrophyte \\
\hline Quercus pyrenaica Willd. & rebollo & 2 & wild & phanerrophyte \\
\hline \multirow[t]{2}{*}{ Quercus spp. } & bellota & 9 & wild & phanerrophyte \\
\hline & roble & 14 & wild & phanerrophyte \\
\hline \multicolumn{5}{|l|}{ Geraniaceae } \\
\hline Pelargonium zonale (L.) L'Hér. ex Aiton & geranio & 3 & decorative & chamaephyte \\
\hline \multicolumn{5}{|l|}{ Iridaceae } \\
\hline Crocus sativus L. & azafrán & 6 & crop & geophyte \\
\hline Iris spp. & lirio & 44 & wild & geophyte \\
\hline \multicolumn{5}{|l|}{ Juglandaceae } \\
\hline Juglans regia L. & nogal & 39 & crop & phanerophyte \\
\hline \multicolumn{5}{|l|}{ Juncaceae } \\
\hline Juncus spp. & junco & 7 & wild & geophyte \\
\hline \multicolumn{5}{|l|}{ Lamiaceae } \\
\hline Lavandula latifolia Medik. & espliego & 4 & wild & chamaephyte \\
\hline Melissa officinalis L. & melisa & 1 & wild & hemicryptophyte \\
\hline Mentha spicata L. & hierbabuena & 27 & crop & hemicryptophyte \\
\hline
\end{tabular}




\begin{tabular}{|c|c|c|c|c|}
\hline Scientific name & Local name & Citations & Presence & Life-form \\
\hline Ocimum basilicum L. & albahaca & 5 & absent & therophyte \\
\hline Rosmarinus officinalis L. & romero & 56 & decorative & phanerophyte \\
\hline Thymus mastichina (L.) L. & mejorana & 1 & wild & chamaephyte \\
\hline Thymus spp. & tomillo & 22 & wild & chamaephyte \\
\hline \multicolumn{5}{|l|}{ Lauraceae } \\
\hline Cinnamomum cassia J.Presl. & canela & 9 & absent & phanerophyte \\
\hline Laurus nobilis L. & laurel & 79 & wild & phenerophyte \\
\hline \multicolumn{5}{|l|}{ Liliaceae } \\
\hline Lilium candidum L. & azucena & 47 & decorative & geophyte \\
\hline \multicolumn{5}{|l|}{ Linaceae } \\
\hline Linum usitatissimum L. & lino & 27 & crop & therophyte \\
\hline \multicolumn{5}{|l|}{ Lythraceae } \\
\hline Punica granatum $\mathrm{L}$. & granado & 2 & crop & phanerophyte \\
\hline \multicolumn{5}{|l|}{ Malvaceae } \\
\hline Gossypium hirsutum L. & algodón & 3 & absent & therophyte \\
\hline Malva sylvestris $\mathrm{L}$. & malva & 5 & wild & hemicryptophyte \\
\hline Theobroma cacao L. & cacao & 14 & absent & phanerophyte \\
\hline \multicolumn{5}{|l|}{ Moraceae } \\
\hline Ficus carica L. & higuera & 29 & crop & phanerophyte \\
\hline Morus spp. & morera & 4 & crop & phanerophyte \\
\hline \multicolumn{5}{|l|}{ Myrtaceae } \\
\hline Syzygium aromaticum (L.) Merr. \& L.M.Perry & clavo & 4 & absent & phanerophyte \\
\hline \multicolumn{5}{|l|}{ Oleaceae } \\
\hline Fraxinus spp. & fresno & 2 & wild & phanerophyte \\
\hline Jasminum fruticans $\mathrm{L}$. & jazmín & 9 & wild & phanerophyte \\
\hline Olea europaea L. & olivo & 114 & crop & phanerophyte \\
\hline \multicolumn{5}{|l|}{ Papaveraceae } \\
\hline Papaver rhoeas L. & amapola & 9 & wild & therophyte \\
\hline \multicolumn{5}{|l|}{ Pinaceae } \\
\hline Cedrus spp. & cedro & 2 & decorative & phanerophyte \\
\hline Pinus halepensis Mill. & pino carrasco & 1 & crop & phanerophyte \\
\hline Pinus pinaster Aiton & pino resinero & 1 & crop & phanerophyte \\
\hline Pinus pinea $\mathrm{L}$. & pino piñonero & 4 & wild & phanerophyte \\
\hline Pinus spp. & pino & 68 & wild & phanerophyte \\
\hline \multicolumn{5}{|l|}{ Piperaceae } \\
\hline Piper nigrum L. & pimienta & 13 & absent & phanerophyte \\
\hline \multicolumn{5}{|l|}{ Poaceae } \\
\hline Arundo donax L. & caña & 5 & absent & geophyte \\
\hline Avena sativa $\mathrm{L}$. & avena & 11 & crop & therophyte \\
\hline Cynodon dactylon (L.) Pers. & grama & 1 & wild & geophyte \\
\hline Hordeum vulgare L. & cebada & 60 & crop & therophyte \\
\hline
\end{tabular}




\begin{tabular}{|c|c|c|c|c|}
\hline Scientific name & Local name & Citations & Presence & Life-form \\
\hline Oryza sativa L. & arroz & 11 & absent & therophyte \\
\hline Phyllostachys spp. & bambú & 2 & decorative & phanerophyte \\
\hline Secale cereale L. & centeno & 24 & crop & therophyte \\
\hline Stipa tenacisima L. & esparto & 7 & wild & chamaephyte \\
\hline Triticum spp. & trigo & 142 & crop & therophyte \\
\hline Zea mays L. & maíz & 2 & crop & therophyte \\
\hline \multicolumn{5}{|l|}{ Primulaceae } \\
\hline Primula veris $\mathrm{L}$. & flor de San José & 2 & wild & hemicryptophyte \\
\hline Primula vulgaris Huds. & primavera & 1 & wild & hemicryptophyte \\
\hline \multicolumn{5}{|l|}{ Rosaceae } \\
\hline Crataegus monogyna Jacq. & espino albar & 21 & wild & phanerophyte \\
\hline Cydonia oblonga Mill. & membrillo & 3 & crop & phanerophyte \\
\hline Malus domestica Borkh. & manzano & 71 & crop & phanerophyte \\
\hline Malus sylvestris (L.) Mill. & camueso & 3 & wild & phanerophyte \\
\hline Prunus armeniaca L. & albaricoquero & 2 & crop & phanerophyte \\
\hline Prunus avium (L.) L. & cerezo & 17 & crop & phanerophyte \\
\hline Prunus cerasus L. & guindo & 14 & crop & phanerophyte \\
\hline Prunus domestica L. & ciruelo & 14 & crop & phanerophyte \\
\hline Prunus dulcis (Mill.) D.A.Webb & almendro & 24 & crop & phanerophyte \\
\hline Prunus persica (L.) Batsch & melocotonero & 7 & crop & phanerophyte \\
\hline Prunus spinosa L. & endrino & 7 & wild & phanerophyte \\
\hline Pyrus communis L. & peral & 50 & crop & phanerophyte \\
\hline Rosa gallica L. & rosa de Jericó & 1 & wild & phanerophyte \\
\hline Rosa spp. & rosal & 351 & decorative & phanerophyte \\
\hline Rubus ulmifolius Schott & zarzamora & 34 & wild & phanerophyte \\
\hline \multicolumn{5}{|l|}{ Rubiaceae } \\
\hline Coffea arabica L. & café & 8 & absent & phanerophyte \\
\hline \multicolumn{5}{|l|}{ Rutaceae } \\
\hline Citrus aurantiifolia (Christm.) Swingle & lima & 1 & absent & phanerophyte \\
\hline Citrus limon (L.) Osbeck & limonero & 63 & crop & phanerophyte \\
\hline Citrus sinensis (L.) Osbeck & naranjo & 64 & crop & phanerophyte \\
\hline Ruta graveolens L. & ruda & 1 & wild & chamaephyte \\
\hline \multicolumn{5}{|l|}{ Salicaceae } \\
\hline Populus alba L. & chopo blanco & 4 & crop & phanerophyte \\
\hline Populus spp. & chopo & 37 & crop & phanerophyte \\
\hline Salix $\times$ fragilis $\mathrm{L}$. & mimbrera & 4 & wild & phanerophyte \\
\hline Salix babylonica L. & saúce Ilorón & 1 & decorative & phanerophyte \\
\hline Salix caprea L. & salguera & 1 & wild & phanerophyte \\
\hline Salix spp. & saúce & 1 & wild & phanerophyte \\
\hline \multicolumn{5}{|l|}{ Solanaceae } \\
\hline \multirow[t]{2}{*}{ Capsicum annuum L. } & guindilla & 5 & crop & therophyte \\
\hline & pimiento & 29 & crop & therophyte \\
\hline
\end{tabular}




\begin{tabular}{|c|c|c|c|c|}
\hline Scientific name & Local name & Citations & Presence & Life-form \\
\hline Hyoscyamus niger L. & beleño & 2 & wild & therophyte \\
\hline Lycopersicon esculentum Mill. & tomate & 12 & crop & therophyte \\
\hline Nicotiana tabacum L. & tabaco & 24 & crop & therophyte \\
\hline Solanum melongena L. & berenjena & 1 & crop & therophyte \\
\hline Solanum tuberosum $\mathrm{L}$. & patata & 21 & crop & geophyte \\
\hline \multicolumn{5}{|l|}{ Taxaceae } \\
\hline Taxus baccata L. & tejo & 1 & wild & phanerophyte \\
\hline \multicolumn{5}{|l|}{ Theaceae } \\
\hline Camellia sinensis (L.) Kuntze & té & 1 & absent & chamaephyte \\
\hline \multicolumn{5}{|l|}{ Ulmaceae } \\
\hline Ulmus minor Mill. & olmo & 11 & wild & phanerophyte \\
\hline \multicolumn{5}{|l|}{ Verbenaceae } \\
\hline Verbena officinalis L. & verbena & 3 & wild & hemicryptophyte \\
\hline \multicolumn{5}{|l|}{ Violaceae } \\
\hline Viola $\times$ wittrockiana Gams & pensamiento & 2 & decorative & hemicryptophyte \\
\hline Viola spp. & violeta & 6 & wild & hemicryptophyte \\
\hline \multicolumn{5}{|l|}{ Vitaceae } \\
\hline Vitis vinifera L. & vid & 197 & crop & phanerophyte \\
\hline \multicolumn{5}{|l|}{ Not defined/[ferns] } \\
\hline & helecho & 2 & wild & geophyte \\
\hline
\end{tabular}

resented in the region), and $8.2 \%$ are decorative (they only appear in gardens, plant pots, or bouquets of flowers). In relation to the number of citations, cultivated plants make up more than half of the citations $(55.1 \%)$, followed by decorative species (23.5\%), wild plants (18.2\%), and absent species (3.2\%). Weeds associated with cereal crops, such as poppy and malva, registered few quotations.

Regarding plant life-forms, $45.4 \%$ species were phanerophytes, $21.1 \%$ were therophytes, $15.8 \%$ were hemicryp- tophytes, $9.2 \%$ were geophytes, and $8.5 \%$ were chamaephytes. Regarding the number of citations for each life-form, the percentage of phanerophytes was highest, representing $60.3 \%$, followed by therophytes (18.3\%) and hemicryptophytes (12.1\%).

Table 2 shows the citation data grouped by botanical family, with 2756 total quotes observed. The number of species that were cited in each family is also mentioned. The plants mentioned correspond to 48 botanical families, and

Table 2. Families mentioned in songbooks of Castilla y León, Spain, noting the number of citations (cites) and species (spp) mentioned.

\begin{tabular}{|c|c|c|c|c|c|c|c|c|}
\hline \multirow[t]{2}{*}{ Families } & \multicolumn{2}{|c|}{ Number } & \multirow[t]{2}{*}{ Families } & \multicolumn{2}{|c|}{ Number } & \multirow[t]{2}{*}{ Families } & \multicolumn{2}{|c|}{ Number } \\
\hline & cites & spp & & cites & spp & & cites & spp \\
\hline Amaranthaceae & 3 & 1 & Burseraceae & 2 & 2 & Fagaceae & 82 & 5 \\
\hline Amaryllidaceae & 48 & 4 & Cannabaceae & 3 & 1 & Geraniaceae & 3 & 1 \\
\hline Apiaceae & 48 & 5 & Caryophyllaceae & 173 & 1 & Iridaceae & 50 & 2 \\
\hline Araliaceae & 11 & 1 & Cistaceae & 1 & 1 & Juncaceae & 7 & 1 \\
\hline Arecaceae & 19 & 2 & Cucurbitaceae & 74 & 4 & Juglandaceae & 39 & 1 \\
\hline Asparagaceae & 1 & 1 & Cupressaceae & 5 & 1 & Lamiaceae & 116 & 7 \\
\hline Asteraceae & 47 & 12 & Cyperaceae & 1 & 1 & Lauraceae & 88 & 2 \\
\hline Betulaceae & 45 & 1 & Ericaceae & 3 & 1 & Liliaceae & 47 & 1 \\
\hline Brassicaceae & 40 & 4 & Fabaceae & 100 & 17 & Linaceae & 27 & 1 \\
\hline
\end{tabular}


Table 2 cont. Families mentioned in songbooks of Castilla y León, Spain, noting the number of citations (cites) and species (spp) mentioned.

\begin{tabular}{|l|c|c|}
\hline \multirow{2}{*}{ Families } & \multicolumn{2}{|c|}{ Number } \\
\cline { 2 - 3 } & cites & spp \\
\hline Lythraceae & 2 & 1 \\
\hline Malvaceae & 22 & 3 \\
\hline Moraceae & 33 & 2 \\
\hline Myrtaceae & 4 & 1 \\
\hline Oleaceae & 125 & 3 \\
\hline Papaveraceae & 9 & 1 \\
\hline Pinaceae & 76 & 5 \\
\hline Piperaceae & 13 & 1 \\
\hline
\end{tabular}

\begin{tabular}{|l|c|c|}
\hline \multirow{2}{*}{ Families } & \multicolumn{2}{|c|}{ Number } \\
\cline { 2 - 3 } & cites & spp \\
\hline Poaceae & 265 & 10 \\
\hline Primulaceae & 3 & 2 \\
\hline Rosaceae & 619 & 15 \\
\hline Rubiaceae & 8 & 1 \\
\hline Rutaceae & 129 & 4 \\
\hline Salicaceae & 48 & 6 \\
\hline Solanaceae & 94 & 7 \\
\hline Taxaceae & 1 & 1 \\
\hline
\end{tabular}

\begin{tabular}{|l|c|c|}
\hline \multirow{2}{*}{ Families } & \multicolumn{2}{|c|}{ Number } \\
\cline { 2 - 3 } & cites & spp \\
\hline Theaceae & 1 & 1 \\
\hline Ulmaceae & 11 & 1 \\
\hline Verbenaceae & 3 & 1 \\
\hline Violaceae & 8 & 2 \\
\hline Vitaceae & 197 & 1 \\
\hline Not defined/[ferns] & 2 & \\
\hline & & \\
\hline Total & 2756 & 150 \\
\hline
\end{tabular}

2 citations allude to ferns but do not provide enough detail to deduce the species. The families that recorded the largest number of citations were Rosaceae (619 citations), Poaceae (265 citations), and Vitaceae (197 citations).

The families with the greatest number of species mentioned in the songs were Fabaceae with 17 species, Rosaceae with 15 species, and Poaceae with 10 species, data that are consistent with their agricultural importance and the weight of this sector in the cultural heritage of this region. We also found families that, although represent-

ed by one single species, had a high number of citations, such as Vitaceae, Caryophyllaceae, and Betulaceae.

It was possible to extract information alluding to the use of the plant for 901 of 2756 plant mentions. We distinguished 67 applications, which have been grouped into 7 different categories. Figure 2 shows the most frequent categories and subcategories. The following uses were specified inter alia: food for different animals; shelter for animals, specifying whether it is a snake, pigeon, or hare; or for wood to create different utensils.

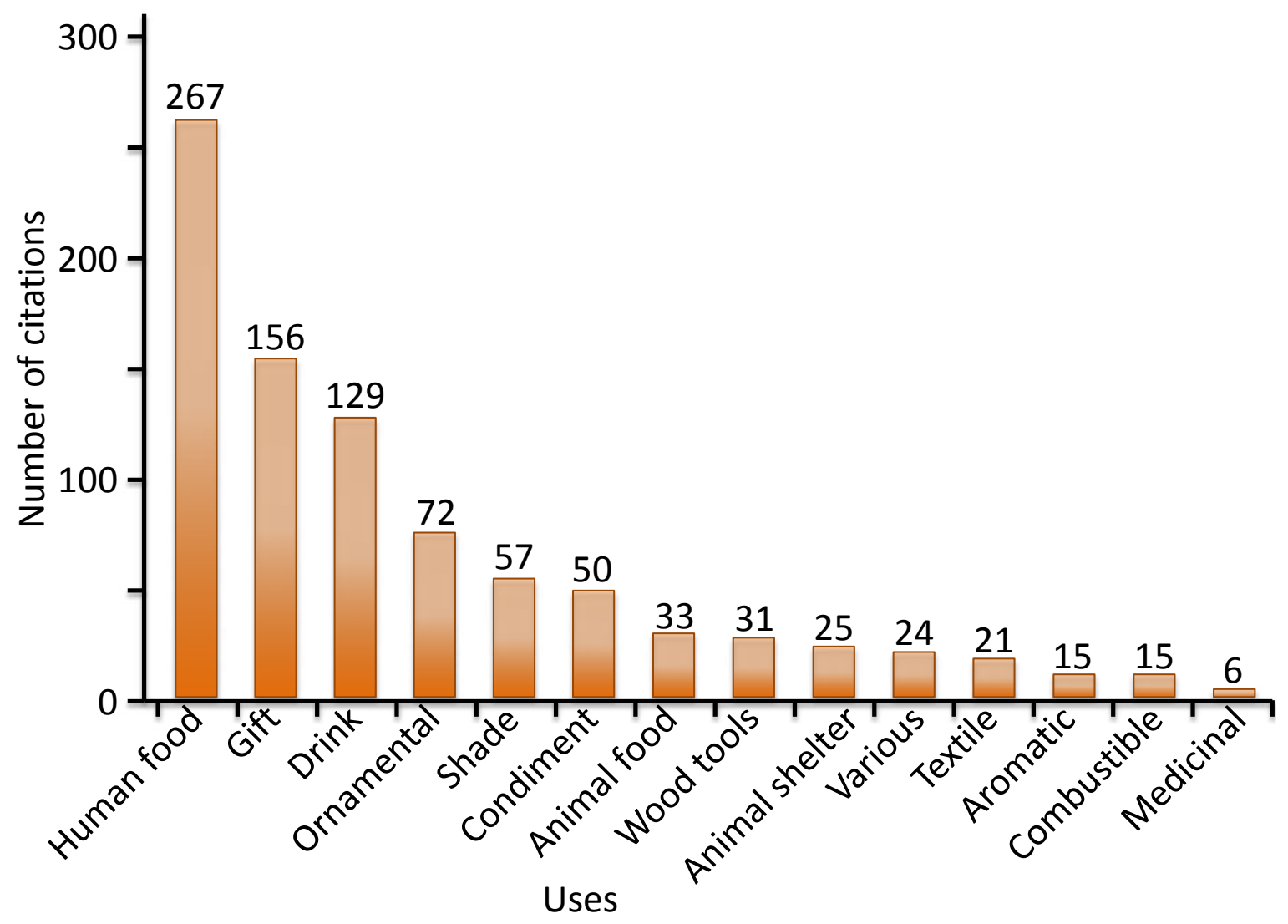

Figure 2. Uses of the plants mentioned in songbooks of Castilla y León, Spain. 
Table 3 shows 35 plants that have 7 or more mentions that allude to their utility. Out of all citations, $32.6 \%$ reflected the usefulness of the plant. The species with the highest number of citations indicating utility are Rosa spp., Vitis vinifera L., Dianthus caryophyllus L., and Triticum spp. As for plants with the greatest diversity of uses, the following were mentioned: Citrus limon (L.) Osbeck, Olea europaea L., and Rosmarinus officinalis L. The other 66 species had less than 7 citations. Utility of the plant was not reflected in 1855 citations. A total of 49 plants were referred to with one single use.
Regarding the part of the plant mentioned, the whole plant (without specifying any part) garnered 976 citations, followed by the fruit and flower, representing $30.2 \%$ and $22.6 \%$, respectively (Figure 3 ). Other parts such as the bark, resin, and seeds were also mentioned, totaling 7 citations. The citations referring to a combination of more than one organ of the plant such as stem-leaf, stem-fruit, or leaf-flower-fruit represent $6.4 \%$.

Table 3. The use of the plants with the highest number of citations (species having 7 or more citations) in the songbooks of Castilla y León, Spain. The table concludes with the number of use citations for these species (Sum) and the total number of citations mentioned for the use in songbooks analyzed (Total).

\begin{tabular}{|c|c|c|c|c|c|c|c|c|c|c|c|c|c|c|c|}
\hline \multirow[t]{2}{*}{ Species } & \multicolumn{15}{|c|}{ Uses } \\
\hline & $\begin{array}{l}\bar{\pi} \\
\frac{\pi}{0} \\
\frac{0}{0} \\
\sum\end{array}$ & 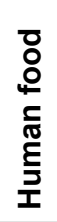 & 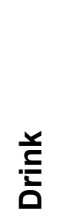 & 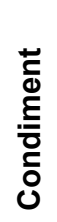 & 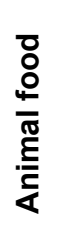 & 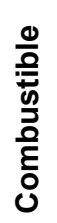 & $\begin{array}{l}\frac{n}{0} \\
0 \\
0 \\
0 \\
0 \\
0 \\
3\end{array}$ & 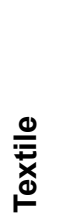 & 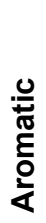 & 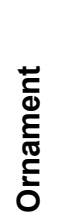 & $\frac{\$}{0}$ & 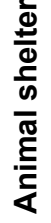 & $\begin{array}{l}\frac{0}{0} \\
\frac{\mathbb{0}}{\infty}\end{array}$ & $\frac{\mathscr{0}}{\frac{0}{2}}$ & 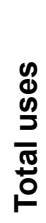 \\
\hline Allium sativum $\mathrm{L}$. & & 8 & & 6 & & & & & & & & & & & 14 \\
\hline Capsicum annuum L. & & 7 & & 4 & & & & & & & & & & & 11 \\
\hline Castanea sativa Mill. & & 14 & & & & & & & & & & & & & 14 \\
\hline Citrus limon (L.) Osbeck & 1 & 6 & 1 & & & & & & 1 & 1 & 6 & 2 & 2 & & 20 \\
\hline Citrus sinensis (L.) Osbeck & 1 & 8 & & & & & & & & & 8 & & 1 & & 18 \\
\hline Corylus avellana $\mathrm{L}$. & & 7 & & & & & & & & & 5 & & & & 12 \\
\hline Crataegus monogyna Jacq. & & 1 & & & & & 1 & & & & & 2 & 6 & & 10 \\
\hline Cucurbita pepo L. & & 8 & & & & & & & & & & & & 1 & 9 \\
\hline Cytisus scoparius (L.) Link & & & & & & & & & 1 & & & 3 & 1 & 2 & 7 \\
\hline Dianthus caryophyllus L. & & & & & & & & & & 13 & 21 & & & & 34 \\
\hline Ficus carica $\mathrm{L}$. & & 8 & & & & & & & & & 1 & 3 & 1 & & 13 \\
\hline Hordeum vulgare L. & & 3 & & & 14 & & & & & & & & & & 17 \\
\hline Iris spp. & & & & & & & & & & 8 & 7 & & & 1 & 16 \\
\hline Juglans regia L. & & 6 & & & & & 11 & & & & 1 & & 1 & & 19 \\
\hline Lactuca sativa L. & & 15 & & & & & & & & & & & & & 15 \\
\hline Laurus nobilis L. & & & & 5 & & & & & & & 9 & & 22 & & 36 \\
\hline Lilium candidum L. & & & & & & & & & 1 & 7 & 3 & & & & 11 \\
\hline Linum usitatissimum L. & & & & & & & & 17 & & & & & & & 17 \\
\hline Malus domestica Borkh. & 1 & 15 & & & & & & & & & 10 & & & & 26 \\
\hline Olea europaea L. & & 2 & & & & 2 & 1 & & & & 2 & 2 & 8 & & 17 \\
\hline $\begin{array}{l}\text { Petroselinum crispum } \\
\text { (Mill.) Fuss }\end{array}$ & & & & 9 & & & & & & & 9 & & & & 18 \\
\hline Phoenix dactylifera L. & & & & & & & & & & 1 & 4 & & 3 & & 8 \\
\hline Pinus spp. & & 2 & & & & & 13 & & & & 1 & 2 & 4 & & 22 \\
\hline Piper nigrum L. & & & & 8 & & & & & & & & & & & 8 \\
\hline
\end{tabular}




\begin{tabular}{|c|c|c|c|c|c|c|c|c|c|c|c|c|c|c|c|}
\hline \multirow[t]{2}{*}{ Species } & \multicolumn{15}{|c|}{ Uses } \\
\hline & 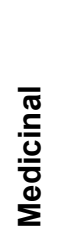 & 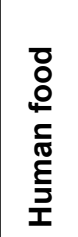 & 訔 & 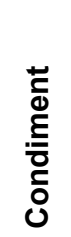 & 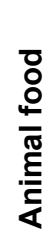 & 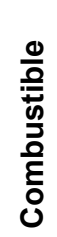 & $\begin{array}{l}\frac{\infty}{0} \\
\stackrel{0}{0} \\
8 \\
\vdots \\
\vdots\end{array}$ & 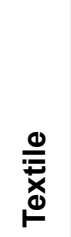 & $\begin{array}{l}0 \\
\stackrel{0}{0} \\
\stackrel{0}{0} \\
\frac{0}{\alpha}\end{array}$ & 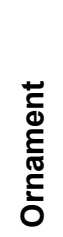 & 志 & 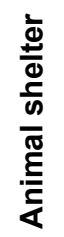 & $\begin{array}{l}\frac{0}{0} \\
\frac{\pi}{\infty} \\
\text { ஸ }\end{array}$ & $\frac{\mathscr{0}}{\frac{0}{0}}$ & 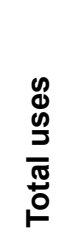 \\
\hline $\begin{array}{l}\text { Prunus dulcis (Mill.) } \\
\text { D.A.Webb }\end{array}$ & & 9 & & & & & & & & 1 & 1 & & & 1 & 12 \\
\hline Pyrus communis L. & & 20 & & & & & & & & & 1 & 1 & & & 22 \\
\hline Quercus ilex L. & & 1 & & & & 7 & & & & & & 2 & & & 10 \\
\hline Rosa spp. & 2 & & & & & & & & 4 & 27 & 37 & & & 2 & 72 \\
\hline Rosmarinus officinalis L. & & & & 1 & & & & & 3 & 1 & 5 & 1 & & 2 & 13 \\
\hline Secale cereale L. & & 11 & & & 2 & & & & & & & & & & 13 \\
\hline Theobroma cacao L. & & 9 & & & & & & & & & 1 & & & 1 & 11 \\
\hline Thymus spp. & & & & & 1 & & & & 3 & 1 & 3 & & & & 8 \\
\hline Triticum spp. & & 24 & & & 10 & & & & & & 1 & & & & 35 \\
\hline Vicia faba $\mathrm{L}$. & & 7 & & & & & & & & & & & & & 7 \\
\hline Vitis vinifera L. & & 7 & 128 & & & & & & & & 2 & & 3 & & 140 \\
\hline Sum & 5 & 198 & 129 & 33 & 27 & 9 & 26 & 17 & 13 & 60 & 138 & 18 & 52 & 10 & 735 \\
\hline Total & 6 & 267 & 129 & 50 & 33 & 15 & 31 & 21 & 15 & 72 & 156 & 25 & 57 & 24 & 901 \\
\hline
\end{tabular}

\section{Discussion}

The songs comprised mostly anonymous poetry, created from the seventeenth to the twentieth century, which were popularized and preserved by oral tradition to the present day (Alín 1991). The number of plants found in this study is similar to that found by Pardo de Santayana et al. (2006) in the work of a single author. In the case of the

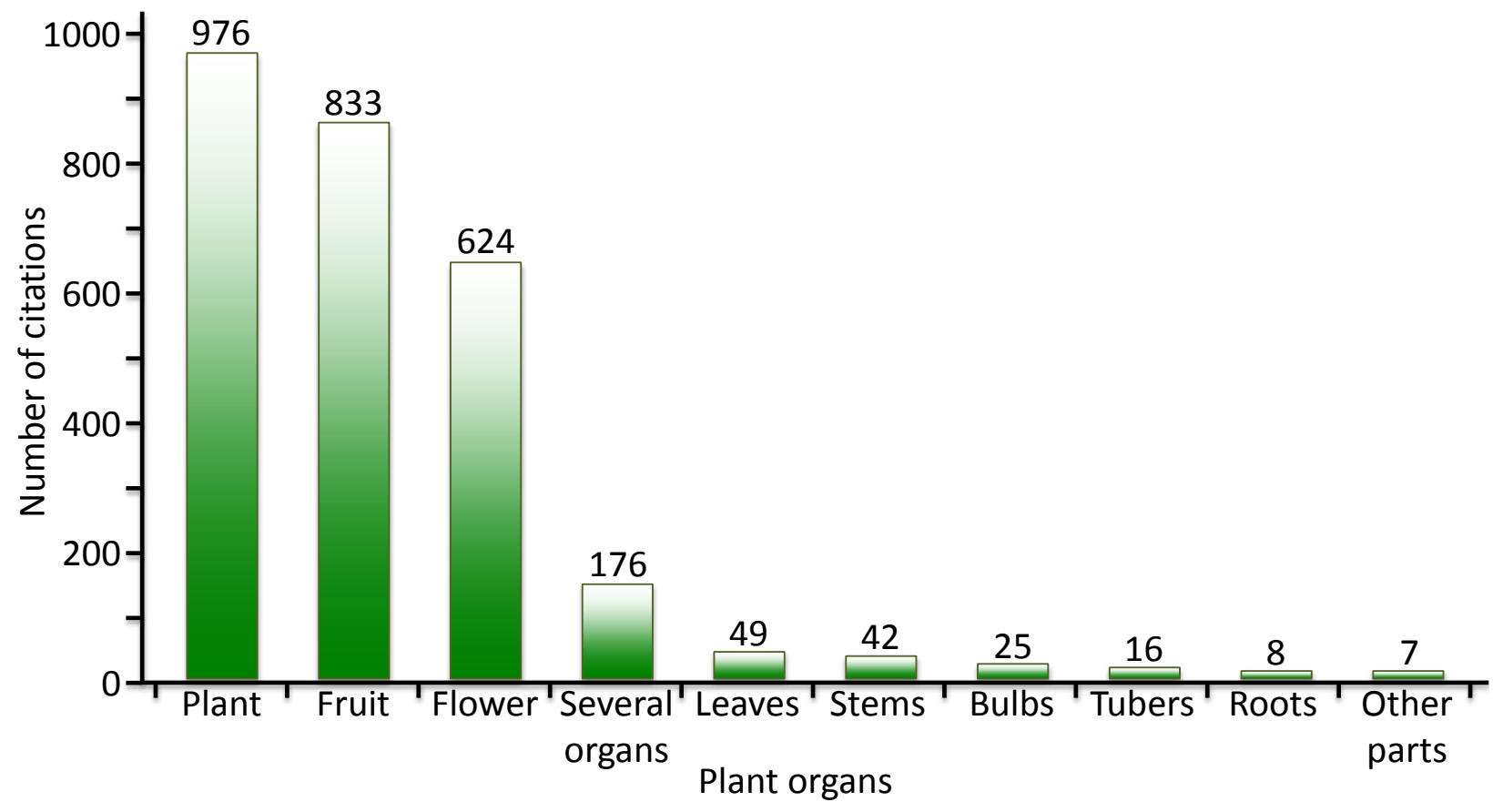

Figure 3. Plant organs referenced in song verses of Castilla y León, Spain. 
songbooks analyzed here, despite the fact that the number of authors is greater, the number of plants mentioned has not increased.

The importance of plants in the economy of rural societies is evident in cultural manifestations such as music, a fact also found in sculpture and painting (Fischer et al. 2011). The high number of citations of crop plants is not surprising, especially in a rural society where cultivated plants are at the center of daily life. Plants associated with crop plants as weeds, which have high representation in paintings and tapestries in the European Mediterranean (Kandeler \& Ullrich 2009), do not appear cited in this songbook.

Exotic species such as frankincense, myrrh, tamarind, cinnamon, clove, palm, royal palm, bamboo, cocoa, and tea are cited in the songbooks, similar to results found by Pardo de Santayana et al. (2006) in a study of ethnobotany in the works of Miguel de Cervantes, which attributes the mention of exotic species to the author's intention to make the literary text more attractive. These species are either mentioned in connection to faraway places, are associated with biblical events, or are provided as reference to the purchasing power of people that have certain spices at their disposal, possibly reminiscent of the ancient spice trade and travel to the Americas. Moldenke \& Moldenke (2002) also highlighted as significant the exotic species found in the Bible.

It is worth noting that, despite representing only $9 \%$ of the plant diversity in the region (Velasco 2009), the presence of phanerophytes and chamaephytes was much higher in the musical texts, comprising $63 \%$ of the plants mentioned, which shows that they are more easily recognized by the region inhabitants.

It is also worth mentioning that the plants that have more relevance to the habits and customs of the region are cited in greater numbers. It is curious that the Rutaceae, with species such as orange, lemon, and lime, has so many mentions given that presence in the community is low. This family also has importance in the work of Cervantes (Pardo de Santayana et al. 2006). Taxaceae, Cupressaceae, and Cistaceae have very few citations despite the fact that they are known colloquially among the people, they are substantial in size, and their recognition is not difficult.

The economic importance of plants in human nutrition and the decorative value of flowers have been highlighted by Rivera and Obón (1991); this is also reflected in the songs studied here. Medicinal plants, however, play only a minor role in song texts, as was also observed by De Cleene and Lejeune (2002). Our findings of the cultural importance of the rose as a gift offering has also been highlighted by other authors (Turner 1988).
Wheat and grapes are staple crops in Mediterranean cultures and diet, and holm oak (Quercus ilex) is one of the most common trees in the landscape of the Iberian Mediterranean ecosystem. The frequency of references of these species is not due to a deliberate choice made by anonymous authors of traditional songs but rather reflects the importance of these plants in everyday life in the region and the general use of plant metaphors in the Spanish language.

The Rosaceae stands out as the plants that are mentioned most for food purposes, followed by Poaceae and Fabaceae, families that are also noted for their nutritional value by Leonti et al. (2006). Aromatic use is represented by plants of the Lamiaceae and Rosaceae, and medicinal use is attributed to plants of the Lamiaceae and Apiaceae, data that coincide with Akerretta et al. (2007).

In terms of alcoholic drinks, the grape (Vitaceae) is mentioned the most, and the Rutaceae (Citrus family) has a prominent role regarding non-alcoholic drinks. The Fagaceae stand out for use as fuel and Salicaceae for use in basket-weaving, data which coincide with other authors (Rivera \& Obón 1991, Velasco 2009). The importance of flowers and fruit confirms their use as gifts and food, uses that are highlighted in other studies (Thompkins \& Bird 1994, Leonti et al. 2006).

\section{Conclusion}

Almost one-fifth of the songs analyzed from the region mentioned plants, a fact that highlights the importance that plants assume in this type of artistic expression. The plants most frequently mentioned in the songs include the rose, grapes, carnation, and wheat, plants that symbolize values of attractiveness and beauty or economic importance due to their agricultural value. The nutritional and decorative uses are the most mentioned, data also corroborated by the fact that the fruits and flowers are the organs with the highest number of citations.

\section{Literature Cited}

Akerreta, S., R.Y. Cavero, V. López \& M.I. Calvo. 2007. Analyzing factors that influence the folk use and phytonomy of 18 medicinal plants in Navarra. Journal of Ethnobiology and Ethnomedicine 3:16-30. dx.doi.org/10.1186/1746$\underline{4269-3-16}$

Alín, J.M. 1991. Cancionero Tradicional. Editorial Castalia, S.A., Madrid, Spain.

Cardaño, M. 2009. Estudio Etnobotánico del Cancionero Popular de Castilla y León. Trabajo Fin de Carrera, Ingeniería Técnica Agrícola, Universidad de Valladolid, Palencia, Spain. 
Causapé, M.C.F. \& F.R. De la Torre. 2009. Un villancico con numerosas plantas medicinales (Santa Fe de Bogotá, Colombia, ca. 1722). Anales de la Real Academia Nacional de Farmacia 75(5):639-678.

Ceballos Jiménez, A. 1986. Diccionario Ilustrado de los Nombres Vernáculos de las Plantas en España. Editorial ICONA, Madrid, Spain.

De Cleene, M. \& M.C. Lejeune. 2002. Compendium of Symbolic and Ritual Plants in Europe. Man \& Culture Publishers, Ghent, Beligum.

Etkin, N.L. \& E. Elisabetsky. 2005. Seeking a transdisciplinary and culturally germane science: The future of ethnopharmacology. Journal of Ethnopharmacology 100(12):23-26. dx.doi.org/10.1016/j.jep.2005.05.025

Fischer, L.P., R. Verilhac, J.J. Ferrandis \& F. Trépardoux. 2011. Les plantes médicinales et symboliques dans les jardins mystiques des retables médiévaux. Histoire des Sciences Medicales 45(3):295-301.

Harris, E.S.J. 2008. Ethnobryology: Traditional uses and folk classification of bryophytes. Bryologist 111(2):169 217. dx.doi.org/10.1639/0007-2745(2008)111[169:ETUA FC]2.0.CO;2

Holman, E.W. 2002. The relation between folk and scientific classifications of plants and animals. Journal of Classification 19(1):131-159.

Kandeler, R. \& W.R. Ullrich. 2009. Symbolism of plants: Examples from European-Mediterranean culture presented with biology and history of art. Journal of Experimental Botany 60(7):1893-1895. dx.doi.org/10.1093/jxb/erp088

Keller, H.A. 2010. Plants used by "guaranies" from Misiones Province (Argentina) to make and improve musical instruments. Darwiniana 48(1):7-16.

Leonti, M., S. Nebel, D. Rivera \& M. Heinrich. 2006. Wild gathered food plants in the European mediterranean: $A$ comparative analysis. Economic Botany 60(2):130-142. dx.doi.org/10.1663/0013-0001(2006)60[130:WGFPIT]2.0 $\mathrm{CO} ; 2$

Mateo, G. \& M. Crespo. 1995. Flora Abreviada de la Comunidad Valenciana. Editorial Gamma, Alicante, Spain.

Moldenke, H.N. \& A.L. Moldenke. 2002. Plants of the Bible. Kegan Paul, London, U.K.

Morales, R., M.J. Macía, E. Dorda \& A. García-Villaraco. 1996. Nombres vulgares, II. Archivos de Flora Ibérica 7:1-325.
Pardo de Santayana, M. 2008. Estudios Etnobotánicos en Campoo (Cantabria). Consejo Superior de Investigaciones Científicas, Madrid, Spain.

Pardo de Santayana, M., J. Tardío, M. Heinrich, A. Touwaide \& R. Morales. 2006. Plants in the works of Cervantes. Economic Botany 60(2):159-181. dx.doi. org/10.1663/0013-0001(2006)60[159:PITWOC]2.0.CO:2

Puri, A.K. \& A. Chaturvedi. 2008. Ethnobotanical approach on wild plants for manufacturing musical instruments by Gond and Koibu tribes of Vidarbha. Indian Journal of Traditional Knowledge 7(1):138-140.

Rivera, D. \& C. Obón. 1991. La Guía Incafo de las Plantas Útiles y Venenosas de la Península Ibérica y Baleares (Excluidas Medicinales). Ed. Incafo, Madrid, Spain.

Ruiz de la Torre, J. 2006. Flora Mayor. Organismo Autonomo Parques Nacionales, Madrid, Spain.

Sánchez, E. 1991. Flora Agrícola. Tomos / y II. Editorial Ministerio de Agricultura, Pesca y Alimentación, Madrid, Spain.

The Plant List. 2013. The Plant List. Version 1.1. www.theplantlist.org Accessed 16 May 2014.

Tropicos. 2014. Tropicos Plant Database. Missouri Botanical Garden. www.tropicos.org Accessed 16 May 2014.

Tompkins, P. \& C. Bird. 1994. The Secret Life of Plants. Harper \& Row, New York, U.S.A.

Turner, N.J. 1988. "The importance of a rose": Evaluating the cultural significance of plants in Thompson and Lillooet Interior Salish. American Anthropologist 90(2):272290. dx.doi.org/10.1525/aa.1988.90.2.02a00020

Velasco, J.M. 2009. Guía de las Plantas Útiles y Perjudiciales en Castilla y León. Editorial Caja Duero, Salamanca, Spain. 
Appendix I. Musical works analyzed from the songbooks of Castilla y León, Spain.

Blanco González, A. 1995. Palabras Contra el Tiempo. Tradición Oral en Peñafiel y Pueblos Adyacentes. Calatrava, Valladolid, Spain.

Caja Segovia. 2003. De la Tierra: Canciones y Romances Segovianos de Ayer y Hoy. Caja Segovia, Segovia, Spain.

Carril Ramos, A. \& M. Manzano Alonso. 1955. Páginas Inéditas del Cancionero de Salamanca. Diputación de Salamanca, Salamanca, Spain.

Cortés Testillano, T. 1991. Cancionero Abulense. Caja de Ávila, Ávila, Spain.

Crespo Alcalde, G. 1991. La Montaña Palentina. Tomo I/ La Lora. Merino Artes Gráficas, Palencia, Spain.

Crespo Alcalde, G. 1991. La Montaña Palentina. Tomo IV Fuentes Carrionas y La Peña. Merino Artes Gráficas, Palencia, Spain.

Díaz, J. 1980. Temas del Cancionero en Castilla y León. Ayuntamiento de Valladolid, Valladolid, Spain.

Díaz, J. 1982. Cancionero del Norte de Palencia I. Institución Tello Téllez de Meneses-Diputación de Palencia, Palencia, Spain.

Díaz, J. 1983. Cancionero de Palencia II. Institución Tello Téllez de Meneses-Diputación de Palencia, Palencia, Spain.

Díaz, J., J. Delfín Val \& L. Díaz de Viana. 1981. Catálogo Folklórico de la Provincia de Valladolid. Vol. IV, cancionero musical primera parte. Institución Cultural Simancas, Valladolid, Spain.

Díaz, J., J. Delfín Val \& L. Díaz de Viana. 1982. Catálogo Folklórico de la Provincia de Valladolid. Vol. IV, cancionero musical segunda parte. Institución Cultural Simancas, Valladolid, Spain.

Díaz de Viana, L. 1993. Música y Culturas. Eudema, Madrid, Spain.

Díaz de Viana, L. \& M. Manzano Alonso. 1989. Cancionero Popular de Castilla y León. Romances, canciones y danzas de tradición oral. Centro de Cultura Tradicional-Diputación de Salamanca, Salamanca, Spain.

Díaz de Viana, L. \& M. Manzano Alonso. 1989. Cancionero Popular de Castilla y León. Romances, canciones y danzas de tradición oral II. Centro de Cultura Tradicional-Diputación de Salamanca, Salamanca, Spain.

Domínguez Berrueta, M. 1941. Del Cancionero Leonés. Diputación de León, León, Spain.

Fernández Núñez, M. 1931. Folklore Leonés. Asilo de Huérfanos del S.C. de Jesús, Madrid, Spain.

García de Andrés, P. \& I. García de Andrés. 2008. Cantos, Rimas y Juegos Populares. Proyecto LIFE Tiermes, Soria, Spain.

Jiménez Juárez, E. 1992. Cancionero Español. Candeleda, Ávila. Manresa, Madrid, Spain.

Manzano Alonso, M. 1991. Cancionero Leonés Vol. II Tomo II. Diputación de León, León, Spain.

Manzano Alonso, M. 1991. Cancionero Leonés Vol. III Tomo I Canto religiosos, ciclo de navidad. Diputación de León, León, Spain.

Manzano Alonso, M. 1992. Cancionero Zamorano. Aeropuerto, Zamora, Spain.

Manzano Alonso, M. 1998. Cancionero Leonés Vol. I Tomo I Ronda y canciones. Diputación de León, León, Spain.

Manzano Alonso, M. 1998. Cancionero Leonés Vol. I Tomo I/ Tonadas y baile. Diputación de León, León, Spain. 
Manzano Alonso, M. 2001. Cancionero Popular de Burgos: Rondas y canciones, Tomo I. Diputación de Burgos, Burgos, Spain.

Manzano Alonso, M. 2001. Cancionero Popular de Burgos: Tonadas de bailes danza, Tomo II. Diputación de Burgos, Burgos, Spain.

Manzano Alonso, M. 2003. Cancionero Popular de Burgos: Canciones infantiles, Tomo IV. Diputación de Burgos, Burgos, Spain.

Manzano Alonso, M. 2003. Cancionero Popular de Burgos: Canciones del ciclo vital y anual, Tomo V. Diputación de Burgos, Burgos, Spain.

Marazuela Bornos, A. 1997. Cancionero de Castilla. Endymión, Segovia, Spain.

Ricis, G. 1981. Obra Musical Palentina del Maestro Guzmán Ricis. Caja Palencia, Palencia, Spain.

Santos, C., L.D. Delgado \& I. Sanz. 1992. Folk Segoviano. I Rueda del año. Caja Segovia, Segovia, Spain.

Santos, C., L.D. Delgado \& I. Sanz. 1998. Folk Segoviano. I La jota segoviana. Caja Segovia, Segovia, Spain.

Sección Femenina. 1936-1975. Fichas de Folklore. Archivo Provincial de Palencia, Palencia, Spain.

Tejero Cobos, I. 1985. Cancionero Popular de Segovia. Gráficas Ceyde, Segovia, Spain. 
